Article

\title{
Inspired to Adopt: The Role of Social Norms in Media Inspiration
}

\author{
Kevin Kryston ${ }^{1, *}$ and Kaitlin Fitzgerald ${ }^{2}$ \\ ${ }^{1}$ Department of Communication, Michigan State University, East Lansing, MI 48824, USA; E-Mail: krystonk@msu.edu \\ 2 Department of Communication, University at Buffalo-State University of New York, Buffalo, NY 14260, USA; \\ E-Mail: ksfitzge@buffalo.edu \\ * Corresponding author
}

Submitted: 30 October 2020 | Accepted: 22 December 2020 | Published: 6 May 2021

\begin{abstract}
We consider the potential for inspirational media content (inspiring videos about dogs) and injunctive norms (social media comments on the videos) to motivate dog adoption behaviors and intentions. In an online experiment, participants were exposed to pretested inspiring (or non-inspiring) videos and social norms cues and were given an opportunity to browse among a series of dogs on a mock adoption website. Participants also indicated their intention to adopt a dog and completed a series of socio-demographic measures. Results indicated that, although both the inspiring videos and the norm cues successfully induced inspiration and perceived injunctive norms, only injunctive norms significantly affected intention to adopt. The effect of norms remained significant when controlling for barriers to adoption such as financial, time, and space considerations. Discussion focuses on implications for inspiring entertainment and social norms theories, and implications for adoptions and other prosocial behaviors.
\end{abstract}

\section{Keywords}

inspirational media; pet adoption; prosocial behavior; selection behavior; social norms

\section{Issue}

This article is part of the issue "The Good, the Bad, and the Ugly: Inspirational Media between Meaning, Narration, and Manipulation" edited by Lena Frischlich (University of Muenster, Germany), Diana Rieger (LMU Munich, Germany) and Lindsay Hahn (University at Buffalo-State University of New York, USA).

(C) 2021 by the authors; licensee Cogitatio (Lisbon, Portugal). This article is licensed under a Creative Commons Attribution 4.0 International License (CC BY).

\section{Introduction}

Heart-warming videos that depict and promote dog adoption are often shared on social media platforms. For instance, videos from "Pittie Nation" - a web series from American media brand, The Dodo-depict pit bulls as kind, lovable dogs to overcome stigma that the breed is dangerous and oftentimes high-needs, and instead, encourage their adoption (see thedodo.com/ series/pittie-nation). Such videos garner considerable attention from social media users in the form of comments, likes, and shares. Naturally, inspirational media content and the social sanctioning of that content on online platforms appear to go hand-in-hand. Thus, there is an increasing need for media effects theories to account for the role of other media users in the effects of inspiring media content.
Some research has begun to examine inspiring media within an online environment (Dale et al., 2020; Janicke, Narayan, \& Seng, 2018; Krämer et al., 2016, 2019; Rieger \& Klimmt, 2019a, 2019b). However, few studies have examined how inspiring media content might influence user attitudes and behaviors, specifically within a social media context (Dale et al., 2020). Even fewer studies have examined the relationship between inspiring content and social norms within a highly controlled, experimental setting (Waddell \& Bailey, 2017). Thus, the current study experimentally tests the effects of inspiring media content when the opinions of others are also present. In particular, we used the context of dog adoption to propose that socially-shared, inspiring dog videos motivate adoption behaviors for three reasons: a) they induce moral elevation, b) they tap into social norms, and c) inspiration and social norms 
may interact such that inspiring media has a greater influence on adoption behaviors when it is considered socially-normative.

First, inspiring media content motivates altruistic behavior by eliciting elevation (Janicke \& Oliver, 2017; Oliver et al., 2018), or the experience of feeling warm and uplifted after viewing acts of moral goodness (Oliver, Hartmann, \& Woolley, 2012). Elevation has been linked to increased prosocial motivation (Ellithorpe, Ewoldsen, \& Oliver, 2015). Dog adoption stories often emphasize the benefits of animal ownership, the altruistic nature of rescuing a dog, and the general kind demeanor of dogs. As such, these videos are emotionally evocative and may induce psychological and physiological elevation that inspires altruistic (i.e., adoption) behaviors (Oliver et al., 2012).

Second, real or perceived social norms moderate the effect of inspiring content. The popularity of these videos on social media makes their consumption a social event. The viewer can see how many people watched the video, and whether reactions to the video were positive or negative. These social signifiers may amplify the effect of inspiration on adoption behaviors such that real or perceived social opinion moderates the motivational effects of inspiration (Krämer et al., 2019; Waddell \& Bailey, 2017). Since inspirational media often facilitates other-focused behaviors (Ellithorpe et al., 2015), normative effects should increase when the viewer is inspired. Research highlighting the sharing of inspiring content via social media (Janicke et al., 2018; Raney et al., 2018) suggests a role of injunctive norms, or a groups' collective approval and sanction of a given behavior (Lapinski \& Rimal, 2005), in moderating inspirational media effects. Dog adoption is generally considered a good deed and something others ought to do (Bir, Widmar, \& Croney, 2018). Thus, injunctive norms likely play a role in motivating viewers' adoption intentions following exposure to the video.

In the current study, we examine the effects of inspiring media content and social norms on dog adoption intentions. Specifically, we examine whether the influences of elevation (induced by an inspiring video about dogs) and norms (communicated as social media comments on the video) motivate dog adoption intentions. We expect that inspiration and injunctive norms will lead to increased adoption intentions, even among those with barriers to dog ownership (including their current financial/living situations and time constraints) and those who previously owned a dog.

We begin with a brief review of the literature on inspirational media and social norms. We then introduce our study and propose study hypotheses grounded in inspirational media and social norms research. We end with a discussion of our findings, specifically highlighting implications for understanding the shared role of inspiring media and social norms in affecting prosocial behaviors like pet adoption.

\subsection{Inspirational Media}

Media researchers have focused recent attention on examining audience interaction with and response to inspirational media content, or content that evokes selftranscendent emotions (Oliver et al., 2018). These experiences evoke emotional responses such as awe, admiration, and elevation. Research on meaningful media experiences initially emerged in contrast to those that are more hedonic or used purely for entertainment and "fun" (Oliver \& Bartsch, 2010). More recently, inspiring media is best understood by the types of content it describes and the simultaneous emotional and motivational responses it evokes in audiences, which we refer to as inspired motivation.' Inspired motivation includes positive emotional responses, psychological and physiological wellbeing, and prosocial motivations (Janicke \& Oliver, 2017).

In particular, we assess the potential for inspiring content to motivate adoption behaviors within a social context. Inspiring media is particularly prevalent on social media platforms such as YouTube and Facebook. In fact, $53 \%$ of American adults report having been inspired by something they saw on social media (Raney et al., 2018; see also Dale et al., 2020). Examining the effects of inspiring media within a social context where social cues are co-present with content cues is therefore a worthwhile endeavor.

Since the effect of social cues can be understood as normative influence (Cialdini, Reno, \& Kallgren, 1990), we apply a social norms framework to make concrete predictions about (1) the direct effects of group influence on adoption behaviors and (2) the way group influence can moderate inspiring media effects.

\subsection{Social Norms}

Social norms refer to a collective code of conduct shared by a referent group (Lapinski \& Rimal, 2005). Individual members of a group gain an understanding of which behaviors are acceptable by observing and interpreting normative information.

There are two types of norms, each with different functions in persuading individuals to adopt normative behavior: 'Descriptive norms' refer to a group's prevalent behaviors (i.e., what most people do) that act as a mental shortcut to quickly make a "correct" decision (Cialdini et al., 1990). 'Injunctive norms' refer to a group's dis/approval or sanction of a given behavior (i.e., permissible behavior for group members). Social rewards and punishments motivate adherence to injunctive norms since failure to adhere to injunctive norms can lead to ostracization (Lapinski \& Rimal, 2005).

As suggested by the Focus Theory of Normative Conduct (Cialdini et al., 1990), individuals formulate normative perceptions (i.e., the perception that others do or think a certain way) by observing social cues in their environment. In this way, people can learn others' norms via the comments, likes, and other social cues associated 
with social entertainment such as YouTube and Facebook videos. In turn, these normative perceptions motivate behavior and behavioral intention, which have potential costs or benefits to other people or society on the whole (Cialdini et al., 1990; Lapinski \& Rimal, 2005). For example, Liu (2017) found that comments indicating approval of water conservation and potential sanctions against water-wasters communicated injunctive norms, and these perceived injunctive norms, in turn, made individuals more likely to adopt pro-water conservation behaviors. Subsequent studies replicated Liu's work in an entertainment context; norms communicated via online social cues (likes, ratings, comments) can lead people to watch "low-quality" films (Kryston \& Eden, 2020) or avoid critically-acclaimed films (Kryston, Park, \& Eden, 2020).

In the context of the current study, we expect that comments indicating approval and sanction of dog adoption, a behavior that can benefit society and the animals themselves, should increase adoption behaviors and intentions, and the effect should be mediated by perceived injunctive norms. Moreover, this effect should persist even among those with barriers to adoption.

Since motivation elicited by inspiring media is associated with socially-oriented cognition (Oliver et al., 2012) that elicits universal orientation (Krämer et al., 2019) and the adoption of prosocial ideals and intentions (Bartsch, Oliver, Nitsch, \& Scherr, 2018, Study 2), we propose that social norms might amplify the effect of inspiration on subsequent prosociality.

Past work found that comments and ratings (i.e., cues indicating social norms) posted on inspiring videos can amplify emotional reactions to inspirational content, including elevation and universal orientation (Krämer et al., 2019). These inspired affective states elicit prosocial motivation, and thus, we expect an additive effect of inspiring content and social norms on adoption behaviors.

\subsection{Current Study}

The current study employs a 2 (Video: inspiring, noninspiring) $\times 3$ (Comments: norm and sanction, comments with no norms, no comment control) between-subjects design. Data were collected in two parts. In part one, we collected socio-demographic data relevant to dog adoption. In part two, participants were randomly assigned to see pretested stimuli and completed measures of adoption behavior and intention to adopt.

\subsubsection{Hypotheses}

Drawing on the aforementioned research on inspiring media and prosocial motivations, we first propose the following hypotheses related to our inspirational video manipulation:

$\mathrm{H1}$ : Viewing an inspiring video will be associated with greater inspired motivation compared to viewing a non-inspiring video;
$\mathrm{H} 2$ : Inspired motivation will be positively associated with adoption behavior $(\mathrm{H} 2 \mathrm{a})$ and intention to adopt (H2b);

H3: There will be an indirect effect of videos on adoption behaviors and intentions through inspired motivation in which those who view an inspiring video will experience greater elevation, and elevation will positively predict adoption behavior $(\mathrm{H} 3 \mathrm{a})$ and intention to adopt (H3b).

Second, drawing on social norms research, we propose the following hypotheses related to our social norm comments manipulation:

H4: Reading approving comments that sanction dog adoption will be associated with higher perceived injunctive norms to adopt a dog compared to (a) reading comments without approval or sanction and (b) videos without comments;

H5: Perceived injunctive norms to adopt a dog will positively influence adoption behavior ( $\mathrm{H} 5 \mathrm{a})$ and intention to adopt ( $\mathrm{H} 5 \mathrm{~b})$;

H6: The effect of comments which sanction dog adoption on adoption behaviors ( $\mathrm{H} 6 \mathrm{a}$ ) and intention to adopt $(\mathrm{H} 6 \mathrm{~b})$ will be mediated by perceived injunctive norms to adopt a dog.

Third, integrating our inspiring video and social norm comments manipulations, we propose the following hypothesis:

H7: There will be a positive interaction between inspired motivation and perceived social injunctive norms on adoption behaviors ( $\mathrm{H} 7 \mathrm{a})$ and intentions (H7b).

Finally, we propose the following hypothesis related to real or perceived barriers to adopt:

H8: The effects of (a) inspired motivation and (b) injunctive norms will remain even when controlling for barriers to adoption.

Since past behavior can be a strong predictor of future behavior (Ouellette \& Wood, 1998), we also wondered if the effects of inspiring media and norms explain adoption behaviors and intention among those who previously owned a dog, leading to the following research question:

RQ1: Will the effects of (a) inspired motivation and (b) injunctive norms remain when controlling for past pet ownership? 


\section{Method}

\subsection{Participants}

Participants $(N=207)$ from a large Midwestern university in the United States were recruited from the communication department participant pool. Data were collected in two parts. Only the data of participants who completed both parts of the study and passed a simple attention check in Part 2 were included in analyses $(n=164)$. Participants received course credit after completing each part. All procedures and stimuli were approved by the host institution's institutional review board.

\subsection{Procedure}

All procedures were completed online using Qualtrics. In Part 1, participants provided informed consent and then completed demographic information, including details about their time commitments, space affordances, and finances. After completing Part 1, participants were invited to participate in Part 2. During this part, participants were randomly assigned to watch one of the four videos, followed by a norms condition. Participants completed a series of measures that assessed their responses to the video and their perceptions of social norms. Then, participants completed the selection task, measures of intention to adopt, and additional demographic information (e.g., gender, age, ethnicity/race).

\subsection{Stimuli}

We conducted a pretest to select videos and comments for our manipulations, as well as photos and descriptions for our selection task. Participants who completed the pretest $\left(n=107 ; M_{\text {age }}=20.05, S D_{\text {age }}=1.80\right)$ were recruited from the same participant pool as the main test and were excluded from the main study.

Participants ranked eight dog descriptions, including eight dog names (four male, four female), ages, and reason for adoption. All dogs were described as being between two- and six-years-old. The results of the pretest indicated minimal variance in dog description ranks $(3.16 \leq M \leq 5.16,1.94 \leq S D \leq 2.39)$; therefore, we selected the middle four descriptions (two male, two female).

Next, participants rated a random set of 10 (out of a possible 16) photos of adult dogs. Examples of each breed identified by Posage, Bartlett, and Thomas (1998) were represented, all the dogs were brown, and the camera angle was held constant for each photo. Photos were rated for dog appeal $(M=5.69, S D=0.97 ; \alpha=0.90)$; the average of nine bipolar scales (e.g., calm-excited, sad-happy), scored from 1 (left word; low appeal) to 7 (right word; high appeal) and how adoptable it was ( $1=$ Not adoptable at all, $7=$ Very adoptable; $M=5.92$, $S D=1.22$ ). Given the left skew and to ensure that our stimuli drove adoption behaviors and intentions rather than the adoptability of the animal itself, we selected dogs lower in appeal and adoptability: chihuahua, pit bull mix, dachshund, and shepherd. These photos were randomly paired to the four selected descriptions.

Three types of videos were pretested: inspiring videos with dogs, informational videos about dogs, and informational videos not about/excluding dogs. Participants were randomly assigned to rate one of the videos $(K=226)$. None of the videos directly advocated for or provided information about dog adoption or ownership.

Participants rated each video using Oliver et al.'s (2012) 15-item emotional elevation $(M=3.64, S D=1.17$; $\alpha=0.98$; e.g., "How much did you feel touched, uplifted?") and 12-item physiological elevation scales $(M=1.90, S D=0.78 ; \alpha=0.89$; e.g., "How often did you feel a lump in throat while watching the video?") were measured on five-point scales. Enjoyment $(M=4.47$, $S D=1.77 ; \alpha=0.95)$ and appreciation $(M=4.70$, $S D=1.77 ; \alpha=0.89$ ) were measured on seven-point scales using Oliver and Bartsch's (2010) three-item measures. Affect $(M=6.47, S D=1.90$; one item where $1=$ sad and $9=$ happy $)$ and arousal $(M=4.45, S D=2.34$; one item where $1=$ bored and $9=$ excited) were measured using the self-assessment mannequin (Bradley \& Lang, 1994). Results indicated that inspiring videos were rated highest in elevation, affect, and appreciation, followed by informational dog videos, and then informational videos not featuring dogs. All pairwise differences were significant.

Thus, we selected exemplar videos from each category: two 'inspiring' videos (one about a labrador, one about a pit bull mix) and two 'non-inspiring' informational videos (one about dogs, one about a baby crib). In the main study, participants were told that a local shelter had posted their assigned video to the shelter's social media feed. A full write-up of pretest results, along with more detailed justification for our decisions, are provided on Open Science Framework (OSF): http://bit.do/ InspiredtoAdopt.

Lastly, to manipulate injunctive norms, we adapted Liu's (2017) stimuli. There were three norms conditions: 'norms,' 'no norms' comments, and a no comment 'control.' In the comment conditions, participants were told that they would see comments posted on the video. The 'norms' comments show approval of dog adoption and potential negative sanctions against those unwilling to adopt. The 'no norms' comments talk about the video content but do not mention adoption. In the 'control' condition, participants were not told they will look at comments and proceeded to complete measures of perceived norms.

\subsection{Measures}

\subsubsection{Mediating Variables}

'Inspired motivation' was measured using a nine-item motivational outcome scale (Oliver et al., 2012; $M=5.66$, 
$S D=0.86$; $\alpha=0.90$; e.g., "I want to be a better person," "I want to do good things for other people"). The scale highly correlates with the physiological and psychological indicators of elevation used in the pretest. Each item was measured on a 7-point scale ( $1=$ Strongly disagree, 7 = Strongly agree). The scale was composed by averaging all of the items.

Perceived injunctive norms were measured using 11 adapted items from Liu's (2017) scale. The scale is usually comprised of three factors-perceived societal approval (e.g., "A majority of people in the United States do not oppose that people adopt a dog"), perceived social approval (e.g., "I feel like most people who are important to me would endorse me adopting a dog"), and perceived social sanction (e.g., "I think people who are important to me would think less of me if I don't adopt a dog")-which are averaged together to create a composite score for perceived injunctive norms. The scale was valid and reliable in past studies (e.g., Kryston et al., 2020). We used the composite score of the 11-item social approval and sanction scales $(M=3.59, S D=0.78$; $\alpha=0.83$ ), referred to as 'perceived social injunctive norms,' since the comments did not manipulate societal approval.

\subsubsection{Dependent Measures}

'Adoption behavior' was measured using a selection task adapted from Knobloch (2003). After exposure to stimuli and completing measures of inspiration and perceived injunctive norms, participants were told that they would be able to look at a webpage featuring dogs listed for adoption at a local shelter.

The screen featured four pretested pictures of dogs presented in random order, along with the option to bypass the selection screen. After clicking a picture, participants were redirected to the dog's profile, which contained pretested descriptions and a short blurb modeled from adoption sites. Participants were allowed to read descriptions for as long as they liked and could stop at any selection screen by clicking the bypass button. After five iterations of dog selections, the selection task automatically ended.

Qualtrics unobtrusively collected whether each participant participated in the dog task $(M=0.92, S D=0.27$, range: $0-1)$, how many dog profiles selected $(M=2.29$, $S D=1.48$, range: $0-5)$, and time spent on dog profiles (in seconds; $M=16.06, S D=14.80$, range: $0-88.71$ ) These three metrics indicated adoption behavior (see Knobloch-Westerwick, 2014).

After the selection task, participants completed an adapted version of the behavioral intent scale found in Park and Smith (2007; $M=4.14, S D=1.84 ; \alpha=0.98$ ) to indicate their 'intention to adopt.' The scale contains four items (e.g., "I have it in my mind to adopt a dog in the near future"). The average of all items was taken to create a scale score for intention to adopt.

\subsubsection{Covariates}

We used barriers to adoption measured in Part 1 as covariates to test $\mathrm{H} 8$ and RQ1: Plans to move ("When do you plan to move from your current residence"; $M=1.36, S D=1.79$; range: $1-7$ where high scores indicate longer stay); weekly free time (in hours; $M=63.53$, $S D=38.94$ ); financial ability to support a dog ("In your opinion, can you currently financially support a dog?"; $M=3.37, S D=2.11$; range: $1-7$ where high scores indicate greater ability to support); independent income $(M=1.45, S D=0.50 ; 1=$ Makes independent income, $2=$ No independent income); and past dog ownership ( $1=$ Yes, $2=$ No; $M=1.32, S D=0.47)$.

All measures and stimuli from the pretest and both parts of the current study can be found on OSF: http://bit.do/InspiredtoAdopt.

\section{Results}

Only 11 participants skipped the selection screen entirely, so effects to profile selection (Yes/No) were not analyzed.

$\mathrm{H} 1-\mathrm{H} 3$ regarded the effect of inspiring videos on adoption behaviors and intention through inspired motivation. To test these hypotheses, we conducted simple mediation (Model 4) with 10,000 bootstrap samples in PROCESS v3.2 (Hayes, 2017). The inspiring video condition was the independent variable (IV) and inspiring motivation was the mediator. The analysis was repeated with number of dog profiles selected, time spent on the selection screen, and intention to adopt as the dependent variables (DV).

At step 1, inspiring video condition significantly predicted inspired motivation $(b=0.43, \mathrm{SE}=0.13$, $p=0.001$ ), indicating that those who saw inspiring videos experienced greater inspired motivation ( $n=88$; $M=5.87, S D=0.72$ ) than those who saw non-inspiring videos $(n=76, M=5.42, S D=0.94)$. Thus, $\mathrm{H} 1$ was supported. At step 2, inspiring affect did not predict number of profiles selected $(b=0.14, \mathrm{SE}=0.14, p=0.31)$, time spent on selection screens $(b=1.64, \mathrm{SE}=1.40, p=0.24)$, or intention to adopt ( $b=0.05, \mathrm{SE}=0.18, p=0.77$ ). The indirect effect was not significant for adoption behaviors or intention (see Figure 1). Thus, $\mathrm{H} 2$ and $\mathrm{H} 3$ were not supported.

$\mathrm{H} 4-\mathrm{H} 6$ regarded the effect of comments on adoption behaviors and intention through perceived norms. We again tested these hypotheses using simple mediation (Model 4) with 10,000 bootstrap samples. Comment condition (multicategorical indicator with control as the comparison group) was the IV and perceived social injunctive norms were the mediator. The test was repeated three times, once for each DV.

At step 1, comment condition significantly predicted perceived social injunctive norms when comparing the norms comment condition to the control $(b=0.38$, $\mathrm{SE}=0.15, p=0.01$ ) but not when comparing the no 


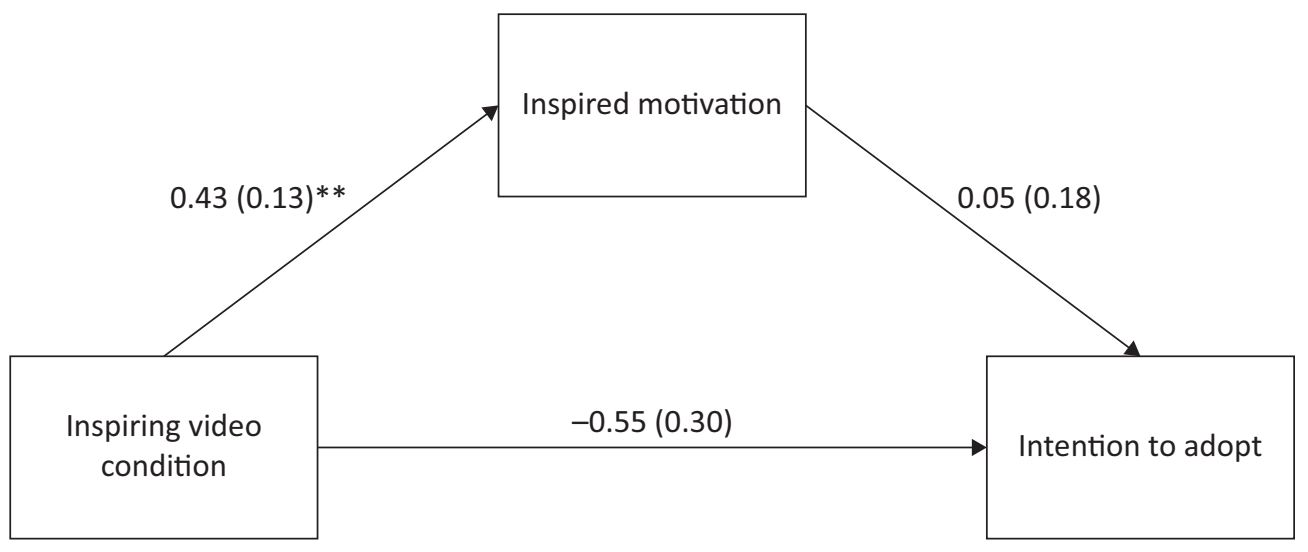

Indirect effect: $0.02(0.08)[-0.15,019]$

Figure 1. Mediated effect of inspiring videos on intention to adopt through inspired motivation. Notes: $*^{*} \leq 0.05, * *$ $p \leq 0.01$. Coefficients associated with each path presented as $b(\mathrm{SE})$. Model statistics: Step $1-F(1,161)=11.14, p=0.001$, adj. $R^{2}=0.06$; Step $2-F(2,160)=1.73, p=0.18$, adj. $R^{2}=0.01$.

norms comment condition to the control $(b=00.17$, $\mathrm{SE}=.15, p=0.26)$. A follow-up analysis of variance (ANOVA) with a post-hoc Bonferroni revealed that perceived social injunctive norms were significantly higher in the norms comment condition ( $n=60, M=3.78$, $S D=0.82)$ than the control $(n=51, M=3.39, S D=0.82)$, but did not differ from the no norms comment condition ( $n=52, M=3.57, S D=0.65 ; F[2,163]=3.38, p=0.04$, $\left.\eta_{2}=0.04\right)$. Thus, $\mathrm{H} 4 \mathrm{~b}$ was supported but $\mathrm{H} 4 \mathrm{a}$ was not.

At step 2, perceived sanctions did not significantly predict number of profile selections $(b=0.20, \mathrm{SE}=0.15$, $p=0.19)$ or time spent $(b=-1.26, \mathrm{SE}=1.48, p=0.40)$. However, perceived social injunctive norms were a significant predictor of intention to adopt $(b=0.59$, $\mathrm{SE}=0.18, p=0.002$ ), indicating that perceptions of others' approval and sanction of adoption elicited stronger intentions to adopt a dog (see Figure 2). Thus, H5b was supported and $\mathrm{H} 5 \mathrm{a}$ was not supported. The indirect effect was significant when comparing the norms com- ment condition to the control $(b=0.22, \mathrm{SE}=0.12, \mathrm{Cl} 95$ $=[0.09,0.48])$, but not when comparing the no norms comment condition to the control $(b=0.10, \mathrm{SE}=0.10$, $\mathrm{Cl} 95=[-0.07,0.31])$. Thus, H6b was partially supported.

$\mathrm{H} 7$ predicted a positive interaction between inspired motivation and perceived social injunctive norms on adoption behaviors and intentions. $\mathrm{H} 7$ was tested using moderated-mediation (PROCESS Model 14; see Figure 3). Given the results of $\mathrm{H} 1-\mathrm{H} 6$, we repeated the tests of $\mathrm{H} 4-\mathrm{H} 6$ regarding the effect of norms, adding inspired motivation to moderate the effect of norms on intention to adopt. At step 1, the effects were the same as reported for $\mathrm{H} 4$. At step 2, main effects of inspired motivation ( $b=0.23, \mathrm{SE}=0.79, p=0.77)$, social injunctive norms ( $b=0.94, \mathrm{SE}=1.14, p=0.41)$, and their interaction $(b=-0.06, \mathrm{SE}=0.21, p=0.76)$ were not significant. Inspired motivation did not moderate the indirect effect of comments on intention through norms. Thus, $\mathrm{H} 7$ was not supported.

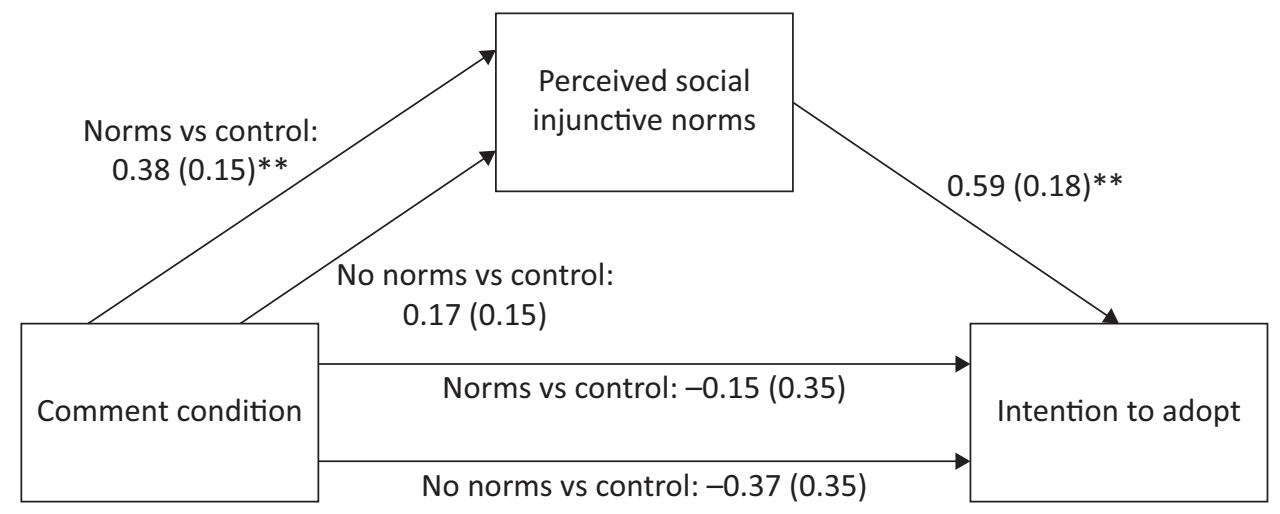

Indirect effect (norms vs control): 0.22 (0.12) [0.03, 0.48]

Indirect effect (no norms vs control): $0.10(0.10)[-0.07,031]$

Figure 2. Mediated effect of comments on intention to adopt through perceived injunctive norms. Notes: ${ }^{*} p \leq 0.05, * *$ $p \leq 0.01$. Coefficients associated with each path presented as $b(\mathrm{SE})$. Model statistics: Step $1-F(2,160)=3.38, p=0.04$, adj. $R^{2}=0.03$; Step $2-F(3,159)=3.24, p=0.02$, adj. $R^{2}=0.05$. 


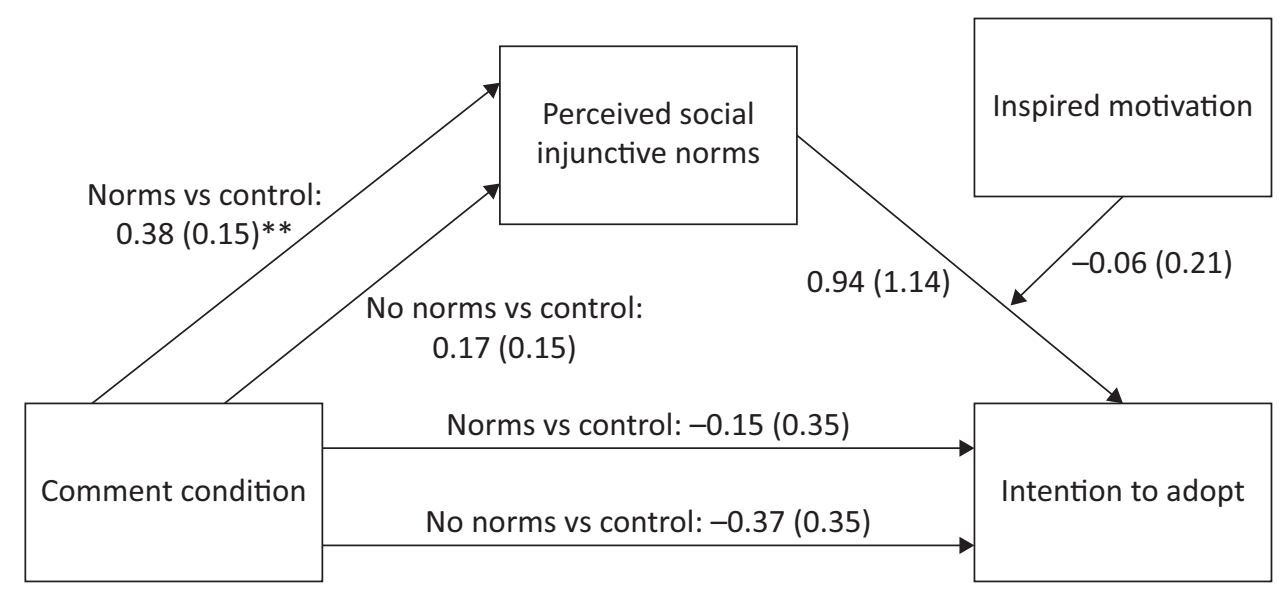

Inspired motivation $\rightarrow$ Intention to adopt: $b=0.23(0.79), p=.77$

Index of moderated mediation (norms vs control): $-0.03(0.10)[-0.22,0.17]$

Index of moderated mediation (no norms vs control): $-0.01(0.06)[-0.15,0.08]$

Figure 3. Moderated-mediation effect of comments on intention to adopt through perceived injunctive norms. Notes: ${ }^{*} p \leq 0.05, * * p \leq 0.01$. Coefficients associated with each path presented as $b(\mathrm{SE})$. Model statistics: Step $1-F(2,160)=3.38$, $p=0.04$, adj. $R^{2}=0.03 ;$ Step $2-F(5,157)=3.27, p=0.05$, adj. $R^{2}=0.04$.

Finally, to test $\mathrm{H} 8$ and answer RQ1, which predicted that the effects of (a) inspired motivation and (b) injunctive norms would remain when controlling for barriers to adoption and past dog ownership, respectively, we conducted hierarchical multiple regression among those who completed all measures across both parts of the study $(n=147)$. Plans to move, weekly free time, financial ability to support a dog, and independent income were entered at step 1 ; inspired motivation and social injunctive norms were entered at step 2; the interaction of inspiration and injunctive norms was entered at step 3. RQ1 was answered by adding past ownership at step 4 . We only conducted an analysis predicting intention to adopt.

At step 1 , independent income was the only significant predictor of intention to adopt $(\beta=-0.18, p=0.03)$, indicating that those who made independent income had a stronger intention to adopt a dog. At step 2 , social injunctive norms $(\beta=0.20, p=0.02)$ and independent income $(\beta=-0.17, p=0.04)$ were the only significant predictors of intention. The interaction term was not significant at step 3 . Thus, $\mathrm{H} 8 \mathrm{~b}$ was partially supported; the effect of perceived social injunctive norms on intention was still significant when controlling for barriers to adoption. H8a was not supported.

Answering RQ1, at step 4, when past ownership was added to the model, not only did past ownership significantly predict intention to adopt $(\beta=-0.19, p=0.03)$, the variable's addition rendered the effect of inspiration, injunctive norms and their interaction not significant. Answering RQ1, those who previously owned dogs showed a greater intention to adopt a dog, and past ownership nullified the effect of inspired motivation and perceived injunctive norms (for additional analyses of barriers to adoption and past ownership, see http://bit.do/ InspiredtoAdopt).

\subsection{Post-Hoc Analysis}

Similar to inspired motivation, inspiration (Krämer et al., 2019) and egoistic motivation (Oliver et al., 2012) can mediate the effect of inspiring media on behavioral outcomes. Thus, the analysis of $\mathrm{H} 1-\mathrm{H} 3$ was repeated four times, once with Oliver et al.'s (2012) 15-item emotional elevation scale ( $M=3.87, S D=0.92 ; \alpha=0.97$ ) and 12-item physiological elevation scale $(M=1.82$, $S D=0.67 ; \alpha=0.86)$, and a five- $(M=6.13, S D=0.94$; $\alpha=0.94$ ) and four-item (item "I would like to do good things for other people" removed; $M=6.08, S D=0.97$; $\alpha=0.93$ ) scales featuring the egoistic (self-focused) items listed in Oliver et al.'s (2012) inspired motivation scale as the mediator.

The results follow a similar pattern to those observed in tests of $\mathrm{H} 1-\mathrm{H} 3$. Although inspiring video condition successfully manipulated emotional and psychological elevation and both versions of the egoistic motivation scale, none of these four variables significantly influenced the number of profiles selected, time spent on selection screens, or intention to adopt, nor did they mediate inspiring media's effect on adoption behavior or intention. Likewise, we tested whether each of these scales significantly interacted with perceived social injunctive norms to predict outcomes of interest (PROCESS Model 1), but all moderation analyses were not significant (see http://bit.do/InspiredtoAdopt for full results).

\section{Discussion}

This study examined the influence of inspiring media and social norms on intentions to adopt a dog. Participants watched a video that was either inspiring or informational (non-inspiring), then read other viewers' 
comments that either sanctioned adoption or did not reference social norms. Those in the control condition did not read comments.

We found that, although both inspiring videos and norm cues successfully induced inspiration and perceived injunctive norms, only norms were significantly associated with adoption intentions. Our study contributes to inspiring media and norms research in a few ways. In particular, we integrate theories from media effects and social psychology to examine how audiences respond to inspiring media content when the opinions of others are present. Our results, including those that were not significant, provide an important addition to the inspiring media and social norms literature which has primarily assessed the effect of online comments on elevation within the context of news media (Waddell \& Bailey, 2017). We discuss this contribution to the literature as well as propose next steps for this research in the following sections.

\subsection{Inspiring Media in a Social Context}

Considerable research has examined the effects of inspiring media when it is experienced alone or in non-social contexts (e.g., Janicke \& Oliver, 2017; Oliver et al., 2018). Far fewer studies have examined the effects of inspiring media while accounting for both the influence of the content and the influence of others' shared opinions about that content. In the current study, we conducted a highly controlled test of inspiring media effects when followed by the shared opinions of others to examine the role of social norms in viewers' response to inspiring media and subsequent prosocial intentions. By including comments that either expressed others' reactions to the inspiring media content or did not reference social norms, we found that others' approval of inspiring media content plays an important role-in this case, the only significant role-in the effects of that content. How the opinions of others influence the effects of inspiring media is an increasingly important question to consider, especially given that inspiring media experiences are so often reported in social contexts (Dale et al., 2020; Raney et al., 2018). Our study contributes to this area of investigation, and we encourage researchers interested in studying inspirational media effects to continue to consider the potential influence of social norms when comments and other social cues are co-present.

Moreover, we examined behavioral intention in two ways: a behavioral proxy (browsing profiles of adoptable dogs; Knobloch-Westerwick, 2014) and self-reported intention to adopt. By including behavioral measures specifically related to content (dog adoption intentions and attitudes), we expand on past research (e.g., Waddell \& Bailey, 2017). Specifically, we found that norms about adoption increased adoption intentions whereas inspirational media about dogs did not. We are careful not to conclude that norms are more powerful motivators than inspiring media. That said, we offer some potential explanations for why only our norms manipulation was influential despite our inspiring content successfully inducing elevation.

First, the fact that we found neither direct nor moderated effects of inspirational media on behavior could raise questions regarding the ability of inspiring media to persuade viewers. Although many studies have observed the effects of inspirational media, these studies tend to examine exposure to inspiring media in a vacuum. For instance, participants view either inspiring or noninspiring media content (e.g., comedy) and indicate whether these media elicit either broad-scale prosocial motivations (e.g., Oliver et al., 2012), or attitude and behavioral changes towards objects that are featured in the inspiring content but not in non-inspiring content (e.g., Krämer et al., 2019). Likewise, outside influences such as social norms or interpersonal interaction are often omitted in related studies (cf. Krämer et al., 2019; Waddell \& Bailey, 2017).

Therefore, other explanations regarding the particular psychological and contextual mechanisms of inspiring media effects should be considered. For example, the Focus Theory of Normative Conduct (Cialdini et al., 1990) and theories of entertainment media effects (e.g., see Tamborini, 2013) suggest that highly-salient media or social cues (that is, cues that are active and accessible in working memory) have a greater influence on behaviors and evaluations than less-salient cues. In the current study, the norm cues: (a) were presented after video exposure (compared to past work which exposed viewers to social cues and inspiring content in tandem; Krämer et al., 2019; Waddell \& Bailey, 2017); (b) explicitly referenced adoption (whereas the videos featured dogs but did not explicitly reference adoption); and (c) advocated a behavior with prosocial implications, which is argued to increase norm salience (Cialdini et al., 1990). Taken together, norm cues may have been more salient than inspiring motivation during the selection task and subsequent measures of intention to adopt, and thus, were more influential for behaviors.

Although our design decisions were purposeful and based on existing literature to test our research questions, these potential explanations for the nonsignificant effect of inspiring media on behavioral outcomes should continue to be examined in future studies. If the norm cues were indeed more salient during the selection task and intention measures, our results could suggest the possibility that the influence of inspiring motivation diminishes when other factors are introduced, or when content is less relevant to the target behavior.

Future work could tackle questions regarding the role of salience in inspiring media effects by: (1) examining the effects of inspiring media over longer periods of time; (2) exploring the psychological processes of inspiring media effects, especially in contexts where social cues are co-present; and (3) examining the extent to which media content, both in terms of the featured topics and moral themes, must match outcomes of interests 
(e.g., would an inspiring film about a family who cannot afford food elicit more donations to a food bank as compared to an inspiring film about an underdog team coming together to win the big game?).

Beyond our contributions to the literature on inspiring media and social norms, our results may also have practical and methodological value. Practically, animal shelters and advocacy organizations may consider using social media content and their followers' approval to improve adoption rates. Methodologically, our study replicated the utility of using a norms scale that captures perceived sanction (Liu, 2017) and a multi-dimensional selection behavior task (Knobloch-Westerwick, 2014). It is also worth noting that the effect of norms on adoption intentions remained significant even when controlling for barriers to adoption, including financial and time considerations. In fact, past dog ownership was the only factor to nullify normative influence on intention to adopt, suggesting that chronically accessible beliefs (Ajzen, 2001) and attitudes (Ewoldsen, Rhodes, \& Fazio, 2015) might have a more powerful influence than contextual norm cues. Still, finding that norms affect adoption intentions despite the presence of financial or time barriers could have implications for instances when pet adoption is not sustainable, such as when an individual lacks the necessary time to care for an adopted dog. In the following section, we consider the potential for negative effects of inspiring media and norms on pet adoption and propose future research to examine this particular circumstance.

\subsection{The Good, the Bad, and the Ugly Effects of Inspiring Media on Pet Adoption}

Dog adoption is typically thought of as a positive behavior, particularly as compared to breeding or purchasing dogs from pet stores. Adoption can reduce euthanasia rates and increase the quality of life for animals in and out of shelters. However, adoption behaviors can also be unsustainable and ultimately destructive for adopters who lack the time and resources to raise an animal, cannot cope with behavioral issues, or are otherwise ill-prepared for pet ownership (Mondelli et al., 2004). In fact, although 1,6 million dogs are adopted each year, approximately $10 \%$ of adopted dogs are rescinded after only six months (American Society for the Prevention of Cruelty to Animals, 2014).

Recent research has considered the seemingly paradoxical potential for inspiring media to motivate behaviors that are ultimately negative or antisocial (i.e., actions that detriment personal or others' well-being; Frischlich, Rieger, Morten, \& Bente, 2019). Indeed, although norms often promote prosocial behaviors (see Shulman et al., 2017, for a review), normative influence can also lead people to perform negative or antisocial behaviors (Reno, Cialdini, \& Kallgren, 1993). For example, terrorist groups use inspirational themes to recruit new members (Frischlich et al., 2019), and individuals are more likely to litter when environmental cues (i.e., litter surrounding a trash can) indicate that most people litter (Cialdini et al., 1990). With relation to the current study, individuals with barriers that reduce the likelihood of successful adoption might be inspired or influenced by norms to adopt pets, even though these individuals may be unable to provide adopted dogs their "forever homes."

Thus, an important follow-up to the current research would be to examine instances when adoption may be problematic: that is, when inspiring media content motivates a behavior that could be positive or negative depending on the individual. To test this, researchers could utilize the methodology of the current study to include an antagonistic injunctive norm condition. In this case, participants would view the inspirational video content (which should motivate pro-adoption intentions), and then read comments which would disapprove of adoption behaviors for individuals without the means to adopt (e.g., "people who don't have time to raise a dog should not adopt"). Additional research might directly compare groups of participants who would be considered as high risk of relinquishing a recently adopted pet based on identified characteristics (e.g. see Mondelli et al., 2004) to those considered low risk of relinquishment.

A similar and interesting direction for future research would be to consider instances when comments communicate disapproval of the inspirational content itself. We assessed norms that were congruent with content (i.e., pro-dog videos and norms). Testing what happens when the content and norms are incongruent warrants investigation. Furthermore, our behavioral outcome (dog adoption) was non-specific; perhaps more powerful effects would be observed if the video and norms advocated specific breeds (e.g., The Dodo's Pittie Nation videos).

Taken together, there are multiple directions for future research to continue to examine the effects of inspiring media on prosocial behaviors and cognitions in social contexts where norms are co-present and salient (e.g., public or online settings). On a final note, we discuss some limitations of the current study.

\subsection{Limitations}

First, we found no effects on dog selection behaviors measured via interaction with our quasi-adoption site. Although we pretested the photos and descriptions, participant engagement may have been low given the limited number of options.

Second, similar to past work by Kryston et al. (2020), perceived sanction was below the scale midpoint, pointing to the difficulty of manipulating the construct. Future studies should consider testing this scale in a survey or develop more powerful sanction manipulations.

Lastly, informational dog videos elicited significantly more inspired motivation than non-dog informational videos. Although combining these videos into a single 
condition still resulted in a successful manipulation of inspired motivation, we caution that content about some subjects (e.g., dogs) may inspire audiences by nature.

\section{Conclusion}

Inspiration and social norms can be powerful motivators, even towards ultimately destructive behaviors. We help to bridge areas of research on the effects of inspiring media when shared on social media platforms where norm cues are present through our methodology and findings. The ways we are influenced by what inspires us - as well as what inspires those around us - continues to be an interesting and important area of communication research.

\section{Acknowledgments}

The authors extend a special thanks to Dr. Allison Eden for supervising this research project.

\section{Conflict of Interests}

The authors declare no conflict of interests.

\section{Supplementary Material}

Supplementary materials are available at http://bit.do/ InspiredtoAdopt.

\section{References}

Ajzen, I. (2001). Nature and operation of attitudes. Annual Review of Psychology, 52(1), 27-58. https:// doi.org/10/cqp7jm

American Society for the Prevention of Cruelty to Animals. (2014). Pet statistics. American Society for the Prevention of Cruelty to Animals. Retrieved from http://www.aspca.org/about-us/faq/pet-statistics

Bartsch, A., Oliver, M. B., Nitsch, C., \& Scherr, S. (2018). Inspired by the Paralympics: Effects of empathy on audience interest in para-sports and on the destigmatization of persons with disabilities. Communication Research, 45(4), 525-553. https://doi.org/10.1177/ 0093650215626984

Bir, C., Widmar, N. O., \& Croney, C. (2018). Exploring social desirability bias in perceptions of dog adoption: All's well that ends well? Or does the method of adoption matter? Animals, 8(9), 154. https://doi.org/ 10.3390/ani8090154

Bradley, M. M., \& Lang, P. J. (1994). Measuring emotion: The self-assessment manikin and the semantic differential. Behavior Therapy and Experimental Psychiatry, 25(1), 49-59. https://doi.org/10.1016/00057916(94)90063-9

Cialdini, R. B., Reno, R. R., \& Kallgren, C. A. (1990). A focus theory of normative conduct: Recycling the concept of norms to reduce littering in public places.
Journal of Personality and Social Psychology, 58(6), 1015-1026. http://doi.org/10.1037/0022-3514.58.6. 1015

Dale, K. R., Raney, A. A., Ji, Q., Janicke-Bowles, S. H., Baldwin, J., Rowlett, J. T., . . . Oliver, M. B. (2020). Selftranscendent emotions and social media: Exploring the content and consumers of inspirational Facebook posts. New Media \& Society, 22(3), 507-527.

Ellithorpe, M. E., Ewoldsen, D. R., \& Oliver, M. B. (2015). Elevation (sometimes) increases altruism: Choice and number of outcomes in elevating media effects. Psychology of Popular Media Culture, 4(3), 236-250.

Ewoldsen, D. R., Rhodes, N., \& Fazio, R. H. (2015). The MODE model and its implications for studying the media. Media Psychology, 18(3), 312-337. http://doi. org/10.1080/15213269.2014.937440

Frischlich, L., Rieger, D., Morten, A., \& Bente, G. (2019). The power of a good story: Narrative persuasion in extremist propaganda and videos against violent extremism. International Journal of Violence and Conflict, 12, 1-16. https://doi.org/10.4119/UNIBI/ijcv. 644

Hayes, A. F. (2017). Introduction to mediation, moderation, and conditional process analysis: A regressionbased approach. New York, NY: Guilford Press.

Janicke, S. H., Narayan, A., \& Seng, A. (2018). Social media for good? A survey on millennials' inspirational social media use. The Journal of Social Media in Society, $7(2), 120-140$.

Janicke, S. H., \& Oliver, M. B. (2017). Meaningful films: The relationship between elevation, connectedness and compassionate love. Psychology of Popular Media Culture, 6(3), 274-289. http://dx.doi.org/ 10.1037/ppm0000105

Knobloch, S. (2003). Mood adjustment via mass communication. Journal of Communication, 53, 233-250. https://doi.org/10.1111/j.1460-2466.2003.tb02588.x

Knobloch-Westerwick, S. (2014). Choice and preference in media use: Advances in selective exposure theory and research. New York, NY: Routledge.

Krämer, N., Eimler, S. C., Neubaum, G., Winter, S., Rösner, L., \& Oliver, M. B. (2016). Broadcasting one world: How watching online videos can elicit elevation and reduce stereotypes. New Media and Society, 19(9), 1349-1368. https://doi.org/10.1177/ 1461444816639963

Krämer, N. C., Neubaum, G., Winter, S., Schaewitz, L., Eimler, S., \& Oliver, M. B. (2019). I feel what they say: The effect of social media comments on viewers' affective reactions toward elevating online videos. Media Psychology. Advance online publication. https://doi.org/10.1080/15213269.2019.1692669

Kryston, K., \& Eden, A. (2020). Examining the effect of social norms on the enjoyment of entertainment. Paper presented at the 70th annual meeting of the International Communication Association, Gold Coast, Australia.

Kryston, K., Park, S., \& Eden, A. (2020). Do social sanc- 
tions explain entertainment avoidance? Paper presented at the 70th annual meeting of the International Communication Association, Gold Coast, Australia.

Lapinski, M. K., \& Rimal, R. N. (2005). An explication of social norms. Communication Theory, 15(2), 127-147. https://doi.org/10.1111/j.1468-2885. 2005.tb00329.x

Liu, W. (2017). The influence of injunctive social norms on food waste prevention behaviors: Toward a culturallybased social normative approach (Unpublished Doctoral Dissertation). Michigan State University, East Lansing, MI, USA.

Mondelli, F., Prato Previde, E., Verga, M., Levi, D., Magistrelli, S., \& Valsecchi, P. (2004). The bond that never developed: adoption and relinquishment of dogs in a rescue shelter. Journal of Applied Animal Welfare Science, 7(4), 253-266. https://doi.org/ 10.1207/s15327604jaws0704_3

Oliver, M. B., \& Bartsch, A. (2010). Appreciation as audience response: Exploring entertainment gratifications beyond hedonism. Human Communication Research, 36(1), 53-81.

Oliver, M. B., Hartmann, T., \& Woolley, J. K. (2012). Elevation in response to entertainment portrayals of moral virtue. Human Communication Research, 38(3), 360-378. https://doi.org/10.1111/j.14682958.2012.01427.x

Oliver, M. B., Raney, A. A., Slater, M., Appel, M., Hartmann, T., Bartsch, A., . . . Das, E. (2018). Selftranscendent media experiences: Taking meaningful media to a higher level. Journal of Communication, 68(2), 380-389. https://doi.org/10.1093/joc/jqx020

Ouellette, J. A., \& Wood, W. (1998). Habit and intention in everyday life: The multiple processes by which past behavior predicts future behavior. Psychological Bulletin, 124(1), 54-74. https://doi.org/10.1037/00332909.124.1.54

Park, H. S., \& Smith, S. W. (2007). Distinctiveness and influence of subjective norms, personal descriptive and injunctive norms, and societal descriptive and injunctive norms on behavioral intent: A case of two behaviors critical to organ donation. Human Communication Research, 33(2), 194-218. https://doi.org/ 10.1111/j.1468-2958.2007.00296.x

Posage, J. M., Bartlett, P. C., \& Thomas, D. K. (1998). Determining factors for successful adoption of dogs from an animal shelter. Journal of the American Veterinary Medical Association, 213(4), 478-482.

Raney, A. A., Janicke, S. H., Oliver, M. B., Dale, K. R., Jones, R. P., \& Cox, D. (2018). Profiling the sources of and audiences for inspiring media content: A national survey. Mass Communication and Society, 21(3), 296-319. https://doi.org/10.1080/15205436. 2017.1413195

Reno, R. R., Cialdini, R. B., \& Kallgren, C. A. (1993). The transsituational influence of social norms. Journal of Personality and Social Psychology, 64(1), 104-112. https://doi.org/10.1037/0022-3514.64.1.104

Rieger, D., \& Klimmt, C. (2019a). The daily dose of digital inspiration: A multi-method exploration of meaningful communication in social media. New Media \& Society, 21(1), 97-118. https://doi.org/10.1177\% 2F1461444818788323

Rieger, D., \& Klimmt, C. (2019b). The daily dose of digital inspiration 2: Themes and affective user responses to meaningful memes in social media. New Media \& Society, 21(10), 2201-2221. https://doi.org/ $10.1177 \% 2 F 1461444819842875$

Shulman, H. C., Rhodes, N., Davidson, E., Ralston, R., Borghetti, L., \& Morr, L. (2017). The state of the field of social norms research. International Journal of Communication, 11, 1192-1213.

Tamborini, R. (2013). Model of intuitive morality and exemplars. In R. Tamborini (Ed.), Media and the moral mind (pp. 43-74). London: Routledge.

Waddell, T. F., \& Bailey, A. (2017). Inspired by the crowd: The effect of online comments on elevation and universal orientation. Communication Monographs, 84(4), 534-550. https://doi.org/10.1080/03637751. 2017.1369137

\section{About the Authors}

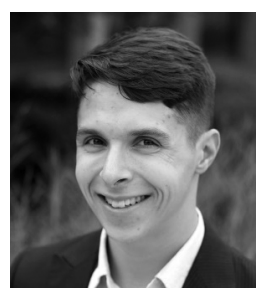

Kevin Kryston (MA, University of Dayton) is a Doctoral Candidate in the Department of Communication at Michigan State University. His research focuses on entertainment media psychology, with a specific focus on social influences on audiences' selection and enjoyment of various forms of entertainment. His work employs behavioral, psychophysiological, and self-report measures to determine the mechanisms underlying audience response.

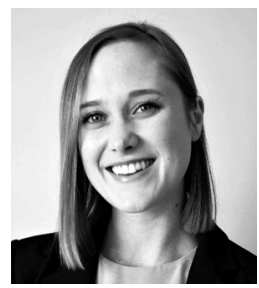

Kaitlin Fitzgerald (MA, University at Buffalo-State University of New York) is a PhD Candidate at the University at Buffalo-State University of New York. Her research examines the cognitive and emotional processes involved in narrative influence and engagement, particularly within the context of entertainment media. 